\title{
Optimization of photon correlations by frequency filtering
}

\author{
Alejandro González-Tudela, ${ }^{1}$ Elena del Valle, ${ }^{2}$ and Fabrice P. Laussy ${ }^{2,3}$ \\ ${ }^{1}$ Max-Planck Institut für Quantenoptik, 85748 Garching, Germany \\ ${ }_{2}^{2}$ Departamento de Física Teórica de la Materia Condensada, Universidad Autónoma de Madrid, 28049 Madrid, Spain \\ ${ }^{3}$ Russian Quantum Center, Novaya 100, 143025 Skolkovo, Moscow Region, Russia
}

(Received 21 December 2014; published 6 April 2015)

\begin{abstract}
Photon correlations are a cornerstone of quantum optics. Recent works [E. del Valle, New J. Phys. 15, 025019 (2013); A. Gonzalez-Tudela et al., ibid. 15, 033036 (2013); C. Sanchez Muñoz et al., Phys. Rev. A 90, 052111 (2014)] have shown that by keeping track of the frequency of the photons, rich landscapes of correlations are revealed. Stronger correlations are usually found where the system emission is weak. Here, we characterize both the strength and signal of such correlations, through the introduction of the "frequency-resolved Mandel parameter." We study a plethora of nonlinear quantum systems, showing how one can substantially optimize correlations by combining parameters such as pumping, filtering windows and time delay.
\end{abstract}

DOI: 10.1103/PhysRevA.91.043807 PACS number(s): 42.50.Ar, 42.50.Lc, 05.10.Gg, 42.25.Kb

\section{INTRODUCTION}

The quantum theory of optical coherence developed by Glauber in the 1960s [1,2] revolutionized the field of quantum optics by identifying photon correlations as the fundamental characterization of light, instead of frequency [3]. This is a great insight since coherence had been understood for centuries as a feature of monochromaticity, while it is now understood in terms of factorizing correlators. This has been confirmed experimentally with the advance of new light sources (such as laser or single-photon sources [4]), as well as progress in photodetection. In the quantum picture, the frequency $v$ of light is linked to the energy $E$ of its constituting particles through Planck constant: $E=h v$. The standard approach of photon correlations has consisted so far essentially in detecting photons from a light source as a function of time, disregarding their frequency. Experimentally, this is achieved either with the original Hanbury Brown-Twiss [5] configuration or by detecting individual photons with a streak camera [6]. For stationary signals, the most popular photon correlation function is the simplest one, correlating two photons. It is known as the second-order correlation function and reads

$$
g^{(2)}(\tau)=\lim _{t \rightarrow \infty} \frac{\left\langle a^{\dagger}(t) a^{\dagger}(t+\tau) a(t+\tau) a(t)\right\rangle}{\left\langle\left(a^{\dagger} a\right)(t)\right\rangle\left\langle\left(a^{\dagger} a\right)(t+\tau)\right\rangle},
$$

with $a(t)$ being the light-field annihilation operator of the system under study at time $t$. If the corresponding spectral shape is singled peak, the question of frequency correlations of the emitted photons may appear a moot point. We will see shortly that it is not. In many cases, nevertheless, the emission is multipeaked, and it is then clear that Eq. (1), which correlates photons regardless of which peak they originate from, is leaving some information out. It is natural to inquire what the correlations of each peak in isolation are or what the cross correlations between peaks are [7,8]. Experimentally, this is readily achieved by inserted filters in the arms of the Hanbury Brown-Twiss configurations [9-13] or using a monochromator in a streak-camera setup [14]. Theoretically, the Glauber correlator must be upgraded to the so-called time- and frequency-resolved photon correlations $[8,15,16]$,

$$
\begin{aligned}
& g_{\Gamma_{1}, \Gamma_{2}}^{(2)}\left(\omega_{1}, \omega_{2} ; \tau\right)
\end{aligned}
$$

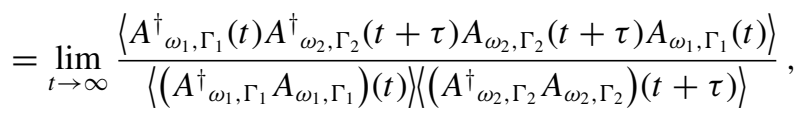

where $A_{\omega_{i}, \Gamma_{i}}(t)=\int_{-\infty}^{t} d t_{1} e^{\left(i \omega_{i}-\Gamma_{i} / 2\right)\left(t-t_{1}\right)} a\left(t_{1}\right)$ is the field detected at frequency $\omega_{i}$, within a frequency window $\Gamma_{i}$, at time $t$. We have recently developed a theory to compute such correlations [17] and introduced the concept of a "two-photon correlation spectrum" (2PS) which, beyond correlating merely peaks, spans over all the possible combinations of photon frequencies $[18,19]$. Landscapes of correlations of unsuspected complexity, which are averaged out in standard photon detection or remain hidden when constraining to particular (fixed) sets of frequencies, are revealed as a result. The 2PS enlarges the set of tools in multidimensional spectroscopy [20-24] and reveals a new class of correlated emission that can be useful for quantum information processing [25,26], for enhancing squeezing [27], or for the study of the foundations of quantum mechanics $[14,28]$. When looking at the full picture put forward by the 2PS, strong correlations turn out to originate from photons which are not part of the spectral peaks. Indeed, a peak results from a single-photon transition between two real states. Various such photons have weak correlations, and even when they do correlate, the correlations are of a classical character. In contrast, collective transitions that require two photons to undertake the emission are strongly and nonclassically correlated since they involve an intermediate virtual state. Only the total energy is fixed, and the photons themselves are emitted at all possible frequencies. When their energies match those of the peaks, stronger single-photon events dominate and spoil the correlations. Away from the peaks, however, the correlated pairs are available in isolation [17-19,25].

Recently, the 2PS of a nontrivial quantum emitter has been experimentally observed [29], with spectacular agreement with the theory and positively identifying, in a rich landscape of correlations, the "leapfrog emission," i.e., an emission between two real states separated by an intermediate virtual one. The violation of Cauchy-Schwarz inequalities by photons emitted 
in such leapfrog processes has also been reported in this work. The emitter was a semiconductor quantum dot, and the physical picture was that of resonance fluorescence in the Mollow-triplet regime [30]. Shortly before that, a 2PS of spontaneous emission was measured from a polariton condensate [14], which features, however, no quantum correlated emission and presents instead a simpler and smooth landscape alternating bunching and antibunching as frequencies become similar or move far apart due to the fundamental Boson indistinguishability. These experiments confirm that the theory is sound and robust and that the physics of photon correlations is ripe to take advantage of the effects uncovered by their tagging with a frequency. For instance, the mere Purcell enhancement of leapfrog processes results in $N$-photon emitters [31].

A central theme of frequency engineering is the interplay between the signal and correlations. Since correlated-emission transiting by virtual states is a high-order process, it is therefore much less frequent than direct emission; that is, the signal is much weaker. This brings up a concern regarding the practical measurement of a 2PS since it requires measuring coincidences from spectral windows where the system already emits very little. Mathematically, this difficulty is concealed for both classes of correlations, Eqs. (1) and (2) alike, by the normalization (denominator) which balances the intensity of the coincidence emission (numerator), turning two vanishing numbers into a finite ratio. In this paper, we address this problem and revisit the 2PS to take into account the available amount of signal. To do so, in Sec. II, we introduce a frequency-resolved Mandel parameter $Q_{\Gamma_{1}, \Gamma_{2}}\left(\omega_{1}, \omega_{2} ; \tau\right)$ that combines both correlations and emission intensity. In light of this parameter, we revisit the two-photon correlation map at $\tau=0$ [19] for several paradigmatic examples in nonlinear quantum optics built around a two-level system. Namely, we consider both its incoherent and coherent driving, the latter bringing the system into the Mollow triplet regime, whose 2PS has already been graced with its experimental observation [29]. We also consider its coupling to a cavity to realize the JaynesCummings (JC) physics, as well as the biexciton configuration found in, typically, quantum-dot systems. These systems are briefly introduced throughout, but mainly to settle notation, and we refer to the literature for the concepts attached to them as well as for their relevance to our problem.

Even the Mandel parameter does not fully capture the problematic of the signal since some correlations are so strong that they dominate over the scarcity of emission. In Sec. III, we complement the information of the available signal with an estimate of the measuring time this supposes, defining a notion of valleys of accessible correlations. There is considerable freedom added by filtering photons when studying their correlations, and we explore diverse ways to optimize them. Various approaches are illustrated for various systems, focusing on the JC model in Sec. IV and the biexciton cascade in Sec. V. In the JC case, we study the optimization with the intrinsic system parameters, namely, the cavity-photon lifetime and the pumping rate, while in the biexciton case, we study the dependence on extrinsic parameters, namely, the filter linewidth and/or time delay. Clearly, a comprehensive analysis could be given along such lines to any system of interest. The present paper aims at illustrating such points in particular cases and leaves it to future works to combine them in the cases where they will be needed.

\section{FREQUENCY-RESOLVED MANDEL PARAMETER}

Mandel introduced a parameter $Q$, which now bears his name, for standard photon correlations (that is, without the frequency information) intended to correct for the previously mentioned normalization issue [32]. The "Mandel parameter" is defined, for a stationary signal, as

$$
Q(\tau)=n_{a}\left[g^{(2)}(\tau)-1\right],
$$

where $n_{a}=\lim _{t \rightarrow \infty}\left\langle a^{\dagger}(t) a(t)\right\rangle$ is the steady-state population of the detected mode. The offset by unity makes the Mandel parameter negative when the light is quantum (in the sense that it is sub-Poissonian and as such has no classical counterpart). The product by $n_{a}$ normalizes the coincidences to the average signal instead of, as done previously, to uncorrelated coincidences. It conveys, therefore, meaningful information on the magnitude of the available signal. Note that $Q(0)=0$ results either from the lack of correlated emission $\left[g^{(2)}(0)=1\right]$ or from too little emission $\left(n_{a} \rightarrow 0\right)$. In this way, the Mandel parameter really characterizes the amount of correlated emission.

Following the spirit of Mandel, we introduce a frequencyresolved version:

$$
\begin{aligned}
& Q_{\Gamma_{1}, \Gamma_{2}}\left(\omega_{1}, \omega_{2} ; \tau\right) \\
& \quad=\sqrt{S_{\Gamma_{1}}^{(1)}\left(\omega_{1}\right) S_{\Gamma_{2}}^{(1)}\left(\omega_{2}\right)}\left[g_{\Gamma_{1}, \Gamma_{2}}^{(2)}\left(\omega_{1}, \omega_{2} ; \tau\right)-1\right],
\end{aligned}
$$

where $S_{\Gamma_{i}}^{(1)}\left(\omega_{i}\right)=\lim _{t \rightarrow \infty}\left\langle\left(A^{\dagger} \omega_{i}, \Gamma_{i} A_{\omega_{i}, \Gamma_{i}}\right)(t)\right\rangle$ is now the steady-state spectrum, which represents, physically, the number of photons passing through the filter of linewidth $\Gamma_{i}$ centered at $\omega_{i}$. Here, it must be emphasized that while $Q(\tau)<$ 0 is a sufficient condition to establish the quantum character of the emission, as it corresponds to a Cauchy-Schwarz inequality (CSI) violation, there is not such a straightforward interpretation for the frequency-resolved version, which would read

$$
\left[g_{\Gamma}^{(2)}\left(\omega_{1}, \omega_{2}, 0\right)\right]^{2}<g_{\Gamma}^{(2)}\left(\omega_{1}, \omega_{1}, \tau\right) g_{\Gamma}^{(2)}\left(\omega_{2}, \omega_{2}, \tau\right) .
$$

Such violation of classical inequalities gives rise to its own landscape of correlations [25]. In contrast, the anticorrelation in frequency, which we will qualify as "frequency antibunching" in agreement with the literature [33], reflects only anticorrelations of intensities, which can be linked or not to a quantum character of the emission.

Our main theme in this paper is illustrated in Fig. 1, starting with the 2PS of the JC [Fig. 1(b)] under weak incoherent pumping in the regime of spontaneous emission [34,35], in which case its spectral line shape is simply the Rabi doublet [Fig. 1(a)]. The physical meaning of this correlation map is amply discussed in Ref. [19]. It is enough for our discussion to highlight the main phenomenology, namely, the set of horizontal and vertical lines that correspond to transitions between real states and the antidiagonal lines $\omega_{1}+\omega_{2}=E_{2, \pm}-E_{0}$ that correspond to two-photon "leapfrog" emission from the second manifold with levels at energies $E_{2, \pm}$ and the ground state at energy $E_{0}$. The transitions at the Rabi frequency $\pm R_{1} \approx \pm g$ 

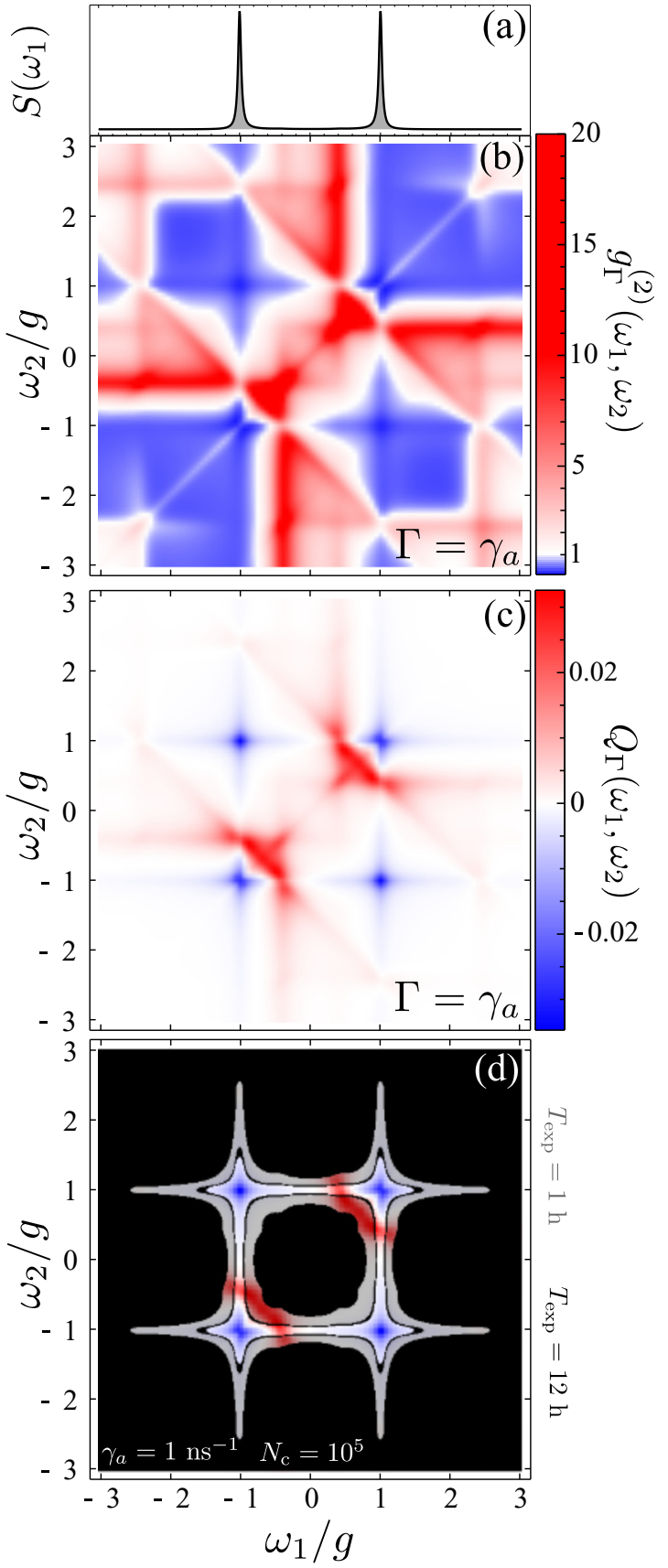

FIG. 1. (Color online) (a) One-photon spectrum for the JC system at low pumping, exhibiting the Rabi doublet of strong coupling. (b) The corresponding 2 PS obtained by spanning $g_{\gamma_{a}}^{(2)}\left(\omega_{1}, \omega_{2}\right)$ over all frequencies. (c) The corresponding frequency-resolved Mandel parameter $Q_{\gamma_{a}}\left(\omega_{1}, \omega_{2}\right)$, retaining only the easily observed features. (d) $Q_{\gamma_{a}}\left(\omega_{1}, \omega_{2}\right)$ with a black (gray) mask superimposed to show only areas where $N_{c} \geqslant 10^{5}$ coincidences are obtained in time $T_{\exp }=12 \mathrm{~h}$ $(1 \mathrm{~h})$ for $\gamma_{a}=1 \mathrm{~ns}^{-1}$. Parameters are $\gamma_{a}=0.1 \mathrm{~g}, \gamma_{\sigma}=0.001 \mathrm{~g}$, and $P_{\sigma}=0.01 \gamma_{a}$.

are all antibunched [blue (dark gray) in Fig. 1] since they are dominated by the decay of one polariton from the lower manifold and one excitation cannot be split into two polaritons, while the lines at $(\sqrt{2} \pm 1) g$ are mainly bunched [red (light gray) in Fig. 1] since they correspond to a cascade from the second manifold. The presence of such cascade correlations in a regime of low excitations, where the second manifold has a vanishing probability to be excited, illustrates the somewhat artificial character of the $2 \mathrm{PS}$. The problem really pertains to photon correlations in general, rather than to the inclusion of frequency, since they similarly predict $g^{(2)}(0)=0$ regardless of the pumping intensity. The same holds for the harmonic oscillator at vanishing pumping, which still generates bunched statistics $g^{(2)}(0)=2$ regardless of the probability to reach two excitations in the system. The paradox arises from the fact that in the limit where the probability of two-photon effects vanishes, so does the possibility to perform a measurement since there is no signal. Instead, if one considers the Mandel correlations, Eq. (4), that are shown in Fig. 1(c), one sees how the result makes more physical sense: most of the nonlinear features have disappeared or are considerably weakened in the regions where there is a strong signal (the correlations tend to die more slowly than the signal), and the remaining features are concentrated on antibunching between the peaks, as well as a trace of the bunching cascades. The leapfrog correlations are extremely strong, which is a general result in all systems, while the antibunching background that dominates the 2PS profile has now disappeared. It is also worth noting how the autocorrelation of each peak (along the diagonal) has a butterfly shape due to indistinguishability bunching enforced by filtering [19], while cross correlation between the two peaks feature a structureless, and therefore neater, antibunching. This could be of interest for single-photon emitters [33].

Although the Mandel correlation spectrum [Fig. 1(c)] appears more physical than the underlying 2PS [Fig. 1(b)], the latter still presents us with a more fundamental physical picture. Indeed, we have merely tamed the features, not removed them, and it is useful to keep track of correlations even though they are out of the reach of an actual experiment. In any case, the 2PS could still be measured ideally and should be regarded as a theoretical limiting case. The 2PS indeed converges to a unique result in the limit of vanishing pumping, thereby defining an unambiguous correlation map, while its Mandel counterpart tends to zero and the relative importance of bunching versus antibunching areas in Fig. 1(c) depends on one's choice of the pumping rate. Finally, it is worth mentioning that at the time of writing, the 2PS of resonance fluorescence has already been measured in its entirety [29], even for a large splitting of the satellite peak in spectral windows with little emission. It seems therefore reasonable that with the ever-increasing technological progress, all fundamental quantum optical emitters, even those with much smaller emission rates, will be likewise characterized.

\section{VALLEYS OF ACCESSIBLE CORRELATIONS}

While $Q_{\Gamma}\left(\omega_{1}, \omega_{2}\right)$ provides a physically sound picture of which regions of the 2PS are the most favorable for observation, it also suffers from its own shortcomings. The arbitrary scale of $Q$ makes it difficult to attach to it a quantitative figure of merit. In this section, we further delineate the valleys of accessible correlations based on a down-to-earth estimate of the numbers of coincidences that can be extracted from the emission.

Assuming no correlations, the possibility of detecting at least one coincidence in a time window $\Delta t$ at frequencies $\omega_{i}$, 
within the frequency windows $\Gamma_{i}$, is given by

$$
p=\left(1-e^{-n_{1}}\right)\left(1-e^{-n_{2}}\right),
$$

where $n_{i}=S_{\Gamma_{i}}\left(\omega_{i}\right) \gamma \Delta t$ represents the number of filtered photons from a source that emits at a rate $\gamma$. For simplicity we always consider symmetric filters in this section, $\Gamma_{1}=\Gamma_{2}$. Using that definition and assuming no further inefficiencies in detection, we can estimate the experimental time required to obtain a given number of random coincidences $N_{c}$ as follows:

$$
T_{\exp }=N_{c} \Delta t / p .
$$

With this, we plot the regions that would be resolved with increasing experimental time $T_{\exp }$. For example, assuming $\gamma_{a}=1 \mathrm{~ns}^{-1}$ as a quantum-dot figure of merit [10], we show in Fig. 1(d) the frequency-resolved Mandel parameter with a mask over the regions for which the number of coincidences is $N_{c}<10^{5}$ for a detection time of $T_{\exp }=1 \mathrm{~h}$ (gray) and $12 \mathrm{~h}$ (black) for $\Delta t=1 / \Gamma$. This shows how the regions with a sizable number of coincidences reduce to those involving at least one peak, as expected. Therefore, a first experimental confirmation of these results could be to keep one branch of the setup on one peak and correlate its input with that of the other branch sweeping the entire spectrum. This should display transitions from antibunching to no correlation, strong bunching, no correlation again, and a weaker antibunching in the autocorrelation due to indistinguishability bunching. For this set of parameters, the experiment would need to run stably for a longer time in order to collect the same amount of signal to observe also the leapfrog processes without intersecting with the peaks. There is a nontrivial interplay of the system parameters that helps or hinders the observation of correlations which will be explored in Sec. IV.

Before moving on to the optimization, we review other examples of nonlinear systems explored in Ref. [19] in light of the frequency-resolved Mandel parameter and the estimated time to resolve it. We start with the most basic system that displays a nontrivial map of correlations, namely, the incoherently pumped two-level system, which we recover by setting $g=0$ in the JC model. The one-photon spectrum of this system is a single Lorentzian peak with broadening $\Gamma_{\sigma}=\gamma_{\sigma}+P_{\sigma}$, as shown in Fig. 2(c). Its two-photon Mandel spectrum shows a butterfly shape of anticorrelation, which is typical of two-level systems [19]. By choosing $\Gamma=\gamma_{\sigma}$ and $\gamma_{\sigma}=0.1 \mathrm{~ns}^{-1}$, the analysis of the measuring time shows that a small region of frequency antibunching with $N_{c}>10^{6}$ would be observed within $1 \mathrm{~h}$, whereas most of the butterfly would be observed within $12 \mathrm{~h}$ for the same threshold of counts. Contrary to naive expectations, if filtering far in the tail of a two-level system, one should indeed observe bunching, though this will be much more difficult to observe experimentally.

Next, we consider a resonant coherent driving of the twolevel system, described by the Hamiltonian $H_{d}=\Omega_{\sigma}\left(\sigma+\sigma^{\dagger}\right)$. In the weak-driving regime, the system has recently been exploited to design ultranarrow single-photon sources [36-40]. In the strong-driving regime, which is the one that we focus on in this paper, the spectrum is the well-known Mollow triplet [30], as shown in Fig. 2(f). Frequency-resolved correlations for this system have been theoretically investigated in the past [16,41-44] and were even measured $[13,45,46]$ before the concept of the 2PS was put forward. But in both theoretical and

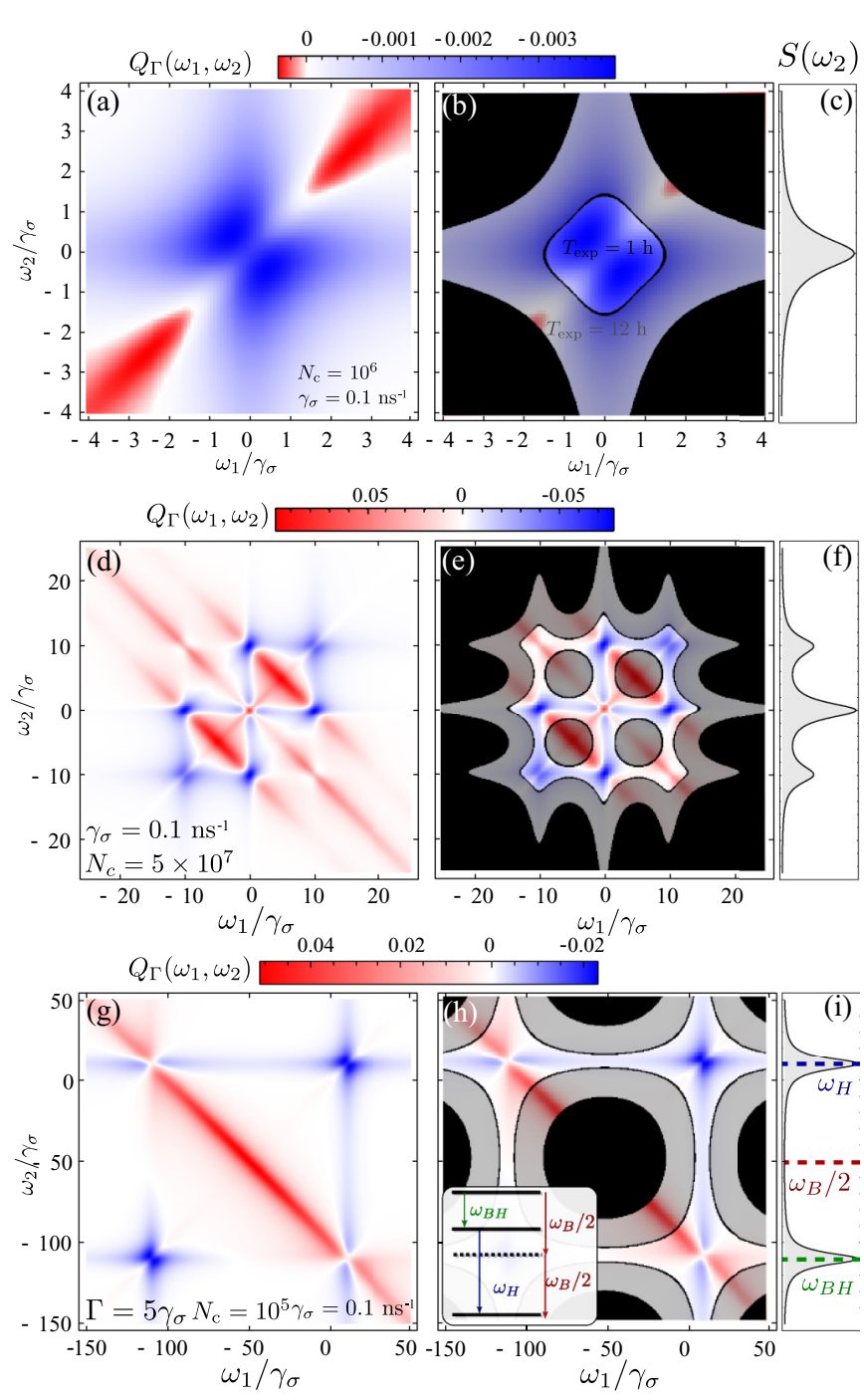

FIG. 2. (Color online) (a) and (b) Mandel two-photon spectra and (c) one-photon spectrum for an incoherently pumped two-level system with $P_{\sigma}=0.01 \gamma_{\sigma}, \gamma_{\sigma}=0.1 \mathrm{~ns}^{-1}$, and $\Gamma=\gamma_{\sigma}$. In (b) we introduce a black (gray) mask for the points where $N_{c}<10^{6}$ in time $T_{\exp }=12 \mathrm{~h}(1 \mathrm{~h})$. (d) and (e) Mandel two-photon spectra and (f) one-photon spectrum for a coherently driven two-level system with $\Omega_{\sigma}=5 \gamma_{\sigma}, \gamma_{\sigma}=0.1 \mathrm{~ns}^{-1}$, and $\Gamma=\gamma_{\sigma}$. In (e) we introduce a black (gray) mask for the points where $N_{c}<5 \times 10^{7}$ in time $T_{\exp }=12$ $\mathrm{h}(1 \mathrm{~h}) .(\mathrm{g})$ and $(\mathrm{h})$ Mandel two-photon spectra and (i) one-photon spectrum for an incoherently pumped biexciton system with $P_{\sigma}=\gamma_{\sigma}$, $\chi=100 \gamma_{\sigma}, \gamma_{\sigma}=0.1 \mathrm{~ns}^{-1}$, and $\Gamma=5 \gamma_{\sigma}$. In (h) we introduce a black (gray) mask for the points where $N_{c}<5 \times 10^{5}$ in time $T_{\text {exp }}=12 \mathrm{~h}$ $(1 \mathrm{~h})$.

experimental contexts, this was at the particular frequencies of the three peaks. However, interesting correlations arise mainly outside the peaks $[19,25]$, at the cost of a weaker signal. This has been confirmed experimentally [29] with the full reconstruction of the Mollow 2PS. Resonance fluorescence is, indeed, ideally suited to pioneer a comprehensive analysis of frequency photon correlations since it is obtained in the strongdriving regime of an extremely quantum emitter, which implies a large emission of strongly correlated photons. Figure 2(e) shows that with $\gamma_{\sigma}=0.1 \mathrm{~ns}^{-1}$ and $\Gamma=\gamma_{\sigma}$, within only $1 \mathrm{~h}$ 

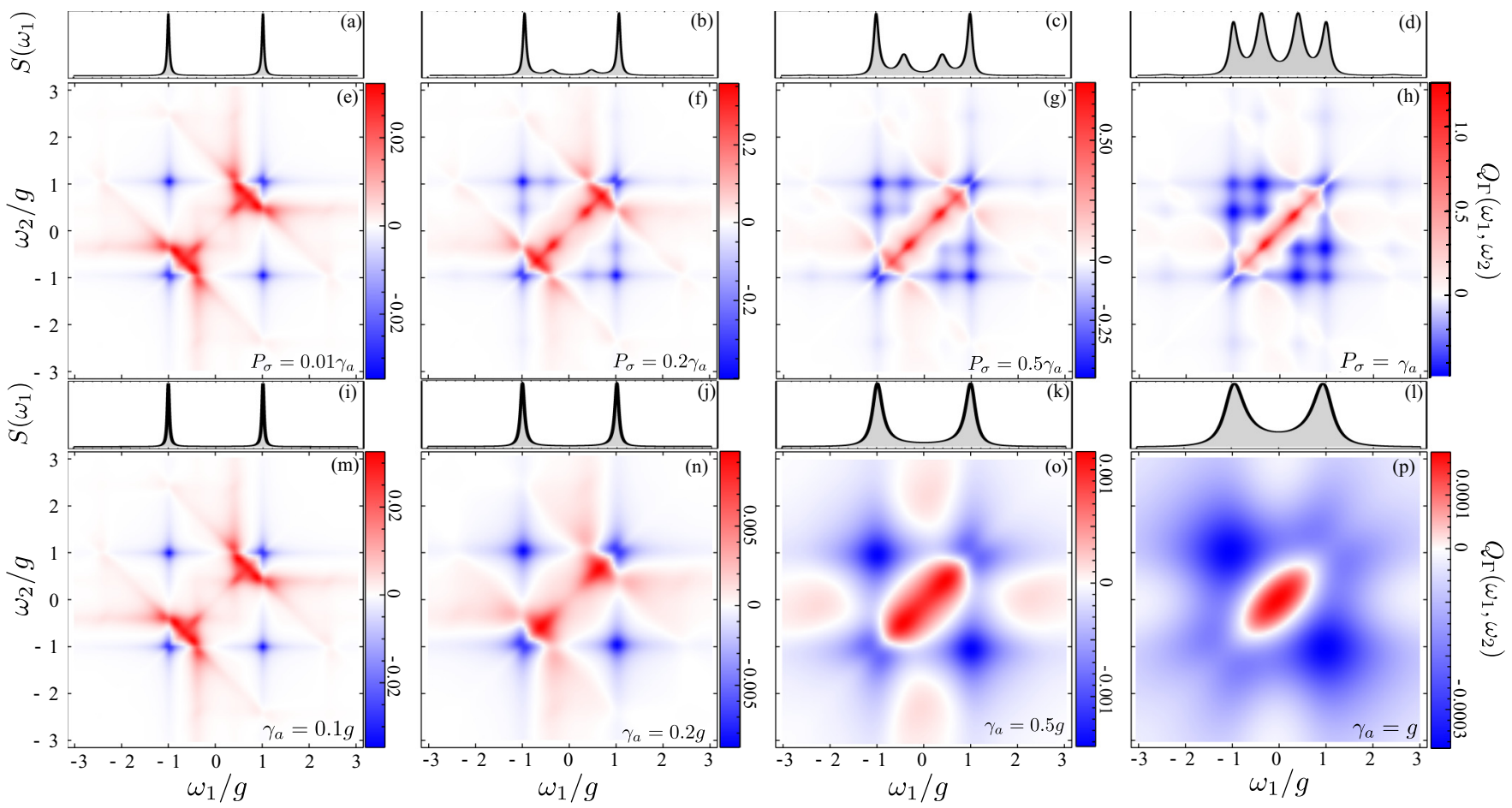

FIG. 3. (Color online) (a)-(d) One-photon spectra for a JC system with $\gamma_{a}=0.1 g, \gamma_{\sigma}=0.001 \mathrm{~g}$, and increasing pumping $P_{\sigma}$ as depicted in the legend. (e)-(h) Mandel two-photon spectra for a JC system with the same parameters as in (a)-(d). (i-l) One-photon spectra for a JC system with $P_{\sigma}=0.01 \mathrm{~g}, \gamma_{\sigma}=0.001 \mathrm{~g}$, and increasing cavity decay rate $\gamma_{a}$ as depicted in the legend. (m)-(p) Mandel two-photon spectra for a JC system with the same parameters as in (i)-(1).

of experimental time, the regions with $N_{c}>5 \times 10^{7}$ unveil the whole horizontal and vertical grid of correlations and a large number of the leapfrogs. It takes only $12 \mathrm{~h}$ to reveal the complete two-photon Mandel spectrum.

Finally, we consider a biexciton level scheme as described in Refs. [18,47,48], which is relevant in semiconductor quantum optics because it describes the typical level structure of quantum dots beyond the simplest two-level-system picture [49-51]. Focusing on a single polarization, it consists of a three-level scheme as depicted in the inset of Fig. 2(h), with a ground state, an excitonic state (at energy $\omega_{H}$ ), and a biexcitonic state whose energy $\left(\omega_{B}\right)$ differs from the sum of its excitonic constituents by $\chi$ due to Coulomb interaction. The one-photon spectrum is then composed of two peaks (at energies $\omega_{H}$ and $\omega_{B H}=\omega_{B}-\omega_{H}$ ) which give rise to an interesting and rich landscape of two-photon correlations. The most prominent feature is the antidiagonal corresponding to the leapfrog between the ground and biexciton states, $\omega_{1}+\omega_{2}=$ $\omega_{B}=-\chi$ (assuming $\omega_{H}=0$ as the reference energy), with the potential for applications in the generation of entangled photon pairs by frequency filtering [18]. With $\gamma_{\sigma}=0.1 \mathrm{~ns}^{-1}$ and setting the threshold at $N_{c}=10^{5}$ random coincidences, within $1 \mathrm{~h}$, the anticorrelation area and bunching of the one-photon transition peaks will be observable, whereas in $12 \mathrm{~h}$ most of its leapfrog structure will be revealed as well, especially by filtering on the sides of the one-photon peaks. Due to both its fundamental importance and practical applications, we will return to the problem of optimizing the observation of the leapfrog processes by changing both the intrinsic parameters and the filtering ones in Sec. V.

\section{OPTIMIZATION OF CORRELATIONS IN THE JAYNES-CUMMINGS MODEL}

We now illustrate how to optimize photon correlations thanks to frequency filtering in the particular case of the JC model. We do a qualitative analysis to avoid focusing the discussion on a particular set of experimental figures of merit. We consider that system parameters are variables for the optimization and defer to the next section (and to other systems) the optimization through extrinsic parameters, e.g., the filters and detection time.

One parameter that can easily be modified is the incoherent pump rate $P_{\sigma}$. The example in the previous section was chosen to be well into the linear regime, i.e., with a very small pumping rate, namely, $P_{\sigma}=0.01 \gamma_{a}$. Increasing pumping has two interesting consequences for the observation of correlations: (1) the signal increases, and (2) the system enters the nonlinear regime.

In Figs. 3(a)-3(h), the effect of increasing pumping is shown for both the spectral shape [Figs. 3(a)-3(d)] and the Mandel parameter resolved in frequency [Figs. 3(e)-3(h)] The spectra let inner peaks appear between the Rabi doublet, corresponding to transitions from the higher manifolds. The corresponding Mandel 2PS also develops new features at the same time the overall intensity of the correlations increases from $\sim 0.02$ with $P_{\sigma}=0.01 \gamma_{a}$ to $\sim 1$ with $P_{\sigma}=\gamma_{a}$ (note the change in the color scales). In particular, the higher manifolds become visible in antibunching only as they get populated, while they manifest themselves in bunching more clearly at low pumping. The Jaynes-Cummings fork provides a well-structured set of correlations between the peaks: the inner 
peaks emit bunched photons but are otherwise antibunched with relation to each other and to the remotest Rabi peak and are uncorrelated with the other, closer, Rabi peak. When various manifolds are well populated, correlations are dominated by real-state transitions, and virtual processes shy away in comparison.

Another parameter that is less easily tuned but that determines the strong-coupling property is the cavity decay rate. In Figs. 3(i)-3(p), the effect of increasing $\gamma_{a}$ is shown, while always keeping the system in the strong-coupling regime $\left(\gamma_{a}<4 g\right)$. This results in the structure smoothing out as well as the intensity of correlations dying (note, again, the color scales). The absolute scale of the Mandel correlations indeed decreases by one order of magnitude, but partly because the cavity gets less populated, and increasing pumping could compensate for that. For $\gamma_{a}=0.5 g$ the leapfrog antidiagonal has disappeared, and for $\gamma_{a}=g$, only the anticorrelation between the Rabi peaks has survived, surrounding a region of indistinguishability bunching. To track more quantitatively how the frequency-resolved correlations evolve with the cavity decay rate, we show in Fig. 4(a) the value of $Q_{\gamma_{a}}$ for pairs of frequencies corresponding to filtering the Rabi peaks. To show that there is some difference due to indistinguishability bunching in the case of autocorrelation, we present the filtering both for the same Rabi peak (solid black line) and for the two Rabi peaks (dashed red line). For $\gamma_{a} \geqslant 4 g$, when the system reaches the weak-coupling regime, frequency-filtered correlations collapse into a single curve since there are no longer polariton modes in the system. It is instructive in this case to plot $g_{\gamma_{a}}^{(2)}\left(R_{1}, \pm R_{1}\right)$ together with the standard $g^{(2)}$ (dotted blue line), as shown in the inset of Fig. 4(a). The difference in this case between filtering the same peak or cross correlating the peaks becomes significant. As already observed, frequency filtering the Rabi peaks improves antibunching relative to nonfiltered correlations, as we discard the frequency regions with bunched photons. This is another instance of how frequency filtering can be used to harness correlations. Note also that worsening the cavity quality factor betters the overall antibunching, while it spoils the peaks antibunching.

\section{OPTIMIZATION OF CORRELATIONS IN A BIEXCITON CASCADE}

We now study photon correlations in a biexciton cascade. To link with the previous discussion on the JC, we show in Fig. 4(b) the dependence on the pumping rate for two configurations, letting the frequency window grow with the pumping as $\Gamma=2\left(\gamma_{\sigma}+P_{\sigma}\right)$. The two configurations are filtering the leapfrog transition at the biexciton frequency (solid black line) and the biexciton-exciton cascade (dashed red line). Both transitions are bunched, $Q_{\Gamma}>0$, due to their two-photon cascade character. However, the one that corresponds to the leapfrog process exhibits a clear optimal pumping intensity at $P_{\sigma} \sim 1.5 \gamma_{\sigma}$, whereas the one-photon transitions exhibit two local maxima at around $P_{\sigma} \sim 0.2 \gamma_{\sigma}$ and $P_{\sigma} \sim 8 \gamma_{\sigma}$ that follow the successive growth of the exciton and biexciton populations. The low pumping regime leads to $Q_{\Gamma} \rightarrow 0$ due to the small emission, whereas in the high-pumping case this is because one recovers the standard photon correlations $g^{(2)}(0) \approx 1$.
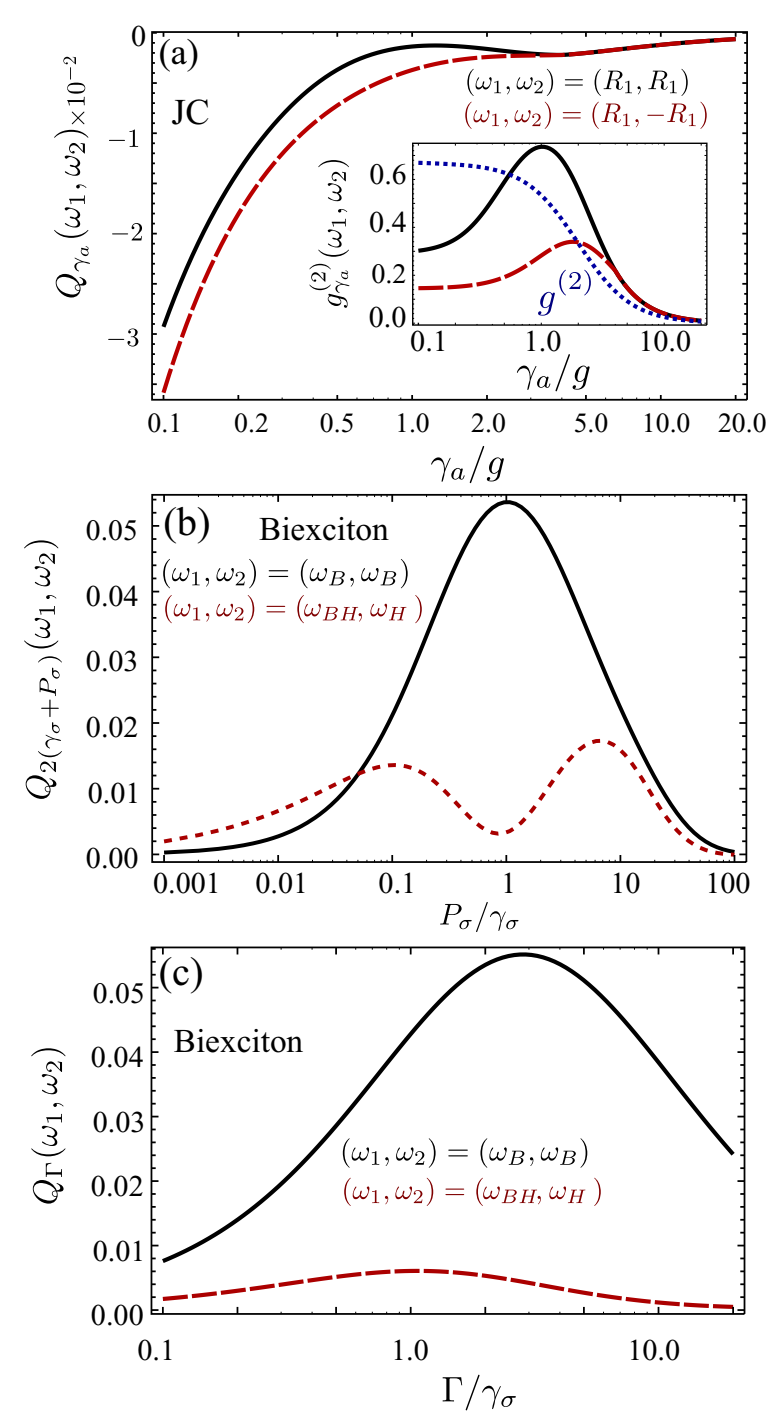

FIG. 4. (Color online) (a) $Q_{\gamma_{a}}\left(\omega_{1}, \omega_{2}\right)$ as a function of $\gamma_{a}$ for a JC system with $P_{\sigma}=0.01 \gamma_{a}, \gamma_{\sigma}=0.001 \mathrm{~g}$ for pairs of frequencies $\left(R_{1}, R_{1}\right)$ (solid black line) and $\left(R_{1},-R_{1}\right)$ (dashed red line). The inset shows $g_{\gamma_{a}}^{(2)}\left(\omega_{1}, \omega_{2}\right)$ for the same parameters, together with standard photon correlation $g^{(2)}(0)$ (dotted blue line). (b) $Q_{\Gamma}\left(\omega_{1}, \omega_{2}\right)$ as a function of $P_{\sigma}$ for a biexciton cascade with the same parameters as in Fig. 2 and $\Gamma=2\left(\gamma_{\sigma}+P_{\sigma}\right)$ for pair a of frequencies as indicated in the inset. (c) $Q_{\Gamma}\left(\omega_{1}, \omega_{2}\right)$ as a function of $\Gamma$, with the same parameters as in Fig. 2.

\section{A. Asymmetric filters}

We now discuss in more detail how the filter linewidth affects the correlations. In Fig. 4(c), we show the dependence on the filter linewidth $\Gamma$ of correlations of the leapfrog cascade (solid black line) and the biexciton-exciton cascade (dashed red line). The leapfrog correlations strongly depend on the filter linewidth due to the virtual nature of such transitions, with an optimum value at around $4 \gamma_{\sigma}$. The biexciton-exciton cascade also displays a maximum $\Gamma \sim \gamma_{\sigma}$, but its dependence is much weaker. In both cases and in contrast to $g_{\Gamma}^{(2)}\left(\omega_{1}, \omega_{2}\right)$ where smaller linewidths optimize leapfrog correlations, the compromise for the signal requires larger filter linewidths to optimize the intensity of correlations. 


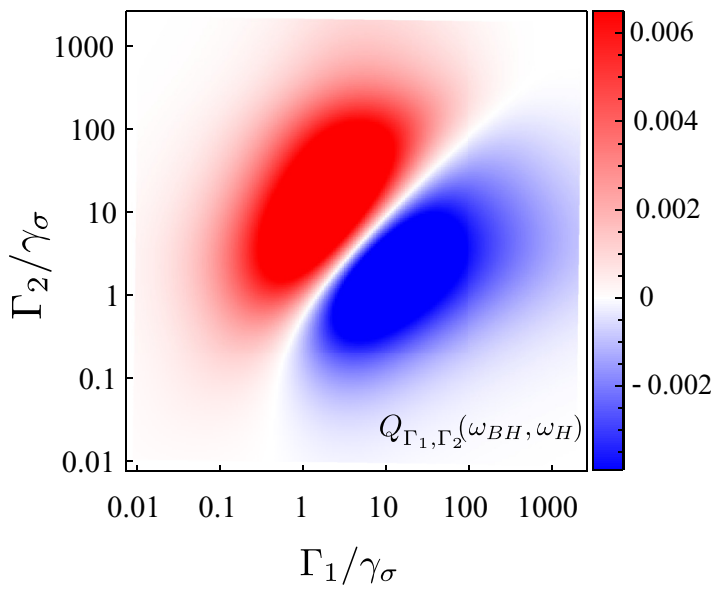

FIG. 5. (Color online) Mandel parameter $Q_{\Gamma_{1}, \Gamma_{2}}\left(\omega_{B H}, \omega_{H}\right)$ when filtering the two peaks of the biexciton-exciton cascade as a function of the widths of the spectral windows. The diagonal corresponds to the typical case of identical filters. Stronger correlations of a varying nature are, however, obtained for asymmetric filters. Parameters are the same as in Fig. 2.

In the analysis so far, we have considered symmetric filters for the two frequencies: $\Gamma_{1}=\Gamma_{2}=\Gamma$. However, for a cascaded emission such as biexciton to exciton to ground state, it is worth exploring the situation where the filters are asymmetric, $\Gamma_{1} \neq \Gamma_{2}$. This is shown in Fig. 5 by filtering the biexciton and exciton peaks for a situation where they have the same broadening and intensity $\left(P_{\sigma}=\gamma_{\sigma}\right)$. Two areas of bunching or antibunching oppose each other depending on the relative value of $\Gamma_{1}$ vs $\Gamma_{2}$, separated by a frontier of no correlation that roughly corresponds to the case of symmetric filters, showing the interest in lifting this limitation even when both spectral peaks are equal. Such a structure is typical of photon cascades. From the level structure, the natural order of the cascade makes it indeed likely we will detect a photon first of frequency $\omega_{B H}$ and then of $\omega_{H}$. Since an $\omega_{B H}\left(\omega_{H}\right)$ photon, filtered with $\Gamma_{1}\left(\Gamma_{2}\right)$, is the first (second) photon in the cascade, if $\Gamma_{2}>\Gamma_{1}$, the time spent by the photon in filter $\omega_{1}$ is larger than the one spent in $\omega_{2}$, which favors the simultaneous detection of the two photons of the cascade and therefore yields a strong bunching. In the opposite regime, when $\Gamma_{1}>\Gamma_{2}$, the $\omega_{B H}$ photon spends less time in the filter, preventing the simultaneous detection of the two photons of the cascade and therefore yielding strong antibunching in the Mandel parameter. There is an optimum value to observe either antibunching or bunching (as a rule of thumb, an order of magnitude difference) since, ultimately, the observations of correlations quench for very broad or asymmetric filters. This loss of correlations is due, interestingly, to the overlap of the filtering windows.

\section{B. Delayed correlations}

We have also restricted our attention to $\tau=0$, i.e., coincidences. However, particularly for cascaded emission, it is to be expected that correlations are maximum at nonzero delay [17]. To condense the bulk of the information into a single figure (Fig. 6), we consider the joint frequency- and time-resolved

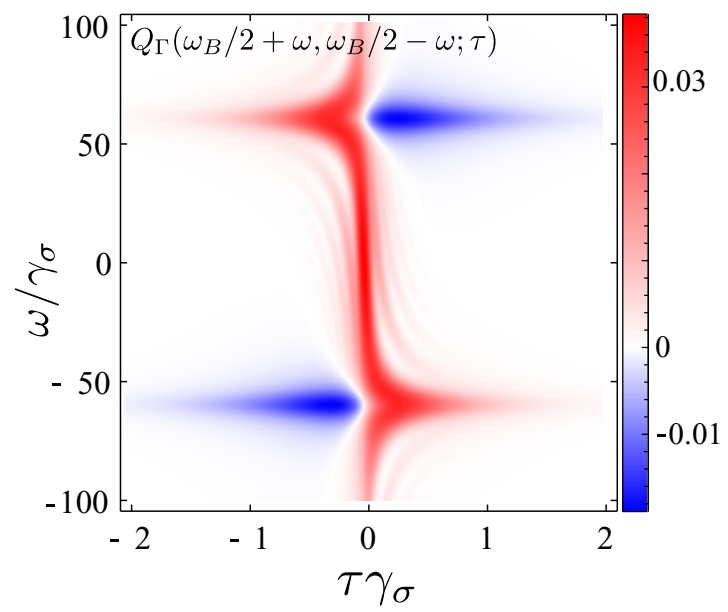

FIG. 6. (Color online) Mandel parameter $Q_{\Gamma}\left(\omega_{B} / 2+\omega\right.$, $\left.\omega_{B} / 2-\omega ; \tau\right)$ when filtering the leapfrog processes (antidiagonal) of the biexciton-exciton cascade and as a function of autocorrelation time. This shows the contrast between leapfrog (virtual) processes, in which the correlations are symmetric in $\tau$ with a fast decay, and real processes, which, when intercepted by the filters, lead to characteristic antibunching-bunching transitions with a slow decay. Parameters are the same as in Fig. 2 except for $\Gamma=10 \gamma_{\sigma}$.

Mandel 2PS along the antidiagonal of Figs. 2(g) and 2(h), $Q_{\Gamma}\left(\omega_{B} / 2+\omega, \omega_{B} / 2-\omega ; \tau\right)$ for $\Gamma=10 \gamma_{\sigma}$. In this line lies the information of both the leapfrogs and the one-photon cascade. Leapfrog emission is maximum at $\omega=0$ [18] and is symmetric in $\tau$, which is typical of second-order processes where the photons, being virtual, have no time order. Due to this symmetry, the optimal delay to observe correlations in this case is $\tau=0$, and correlations decay with the filter time scale $1 / \Gamma$. Contrarily, the biexciton-exciton photon cascade, appearing at $\omega=\left\{\omega_{B H}, \omega_{H}\right\}$, is strongly asymmetric, as clearly shown in Fig. 6. It shows a transition from bunching to antibunching, where there is a definite temporal order in the emission. In this case, the optimal delay to observe strong bunching or antibunching is $\tau \sim 1 / \Gamma$, and the correlations ultimately decay in the intrinsic time scale of the system given by $1 / \gamma_{\sigma}$. The different time scales at which correlations survive between leapfrog and normal cascaded emissions are another consequence of their different physical origins.

\section{Combining the parameters}

Finally, after having explored separately both the dependence on the filter linewidth $\Gamma$ and the temporal delay of the photons $\tau$, one can naturally think of combining them to optimize correlations cumulatively. In Fig. 7, we show the joint $\tau$ and $\Gamma$ dependence of the frequency-resolved Mandel parameter for the two most relevant cases still in the biexciton problem: the simultaneous leapfrog two-photon emission in Fig. 7(a) and the consecutive one-photon transitions in Fig. 7(b). As previously discussed, the temporal shape of the leapfrog process is a symmetric decay of correlations within time scale $1 / \Gamma$, as shown in Fig. 7. As $\Gamma$ increases, so does the decay rate of the correlations [the correlation time and the filter linewidth are roughly in inverse proportion, as shown by the dotted lines, which are $\left.1 /\left(\Gamma / \gamma_{\sigma}\right)\right]$. The $\tau=0$ correlation 


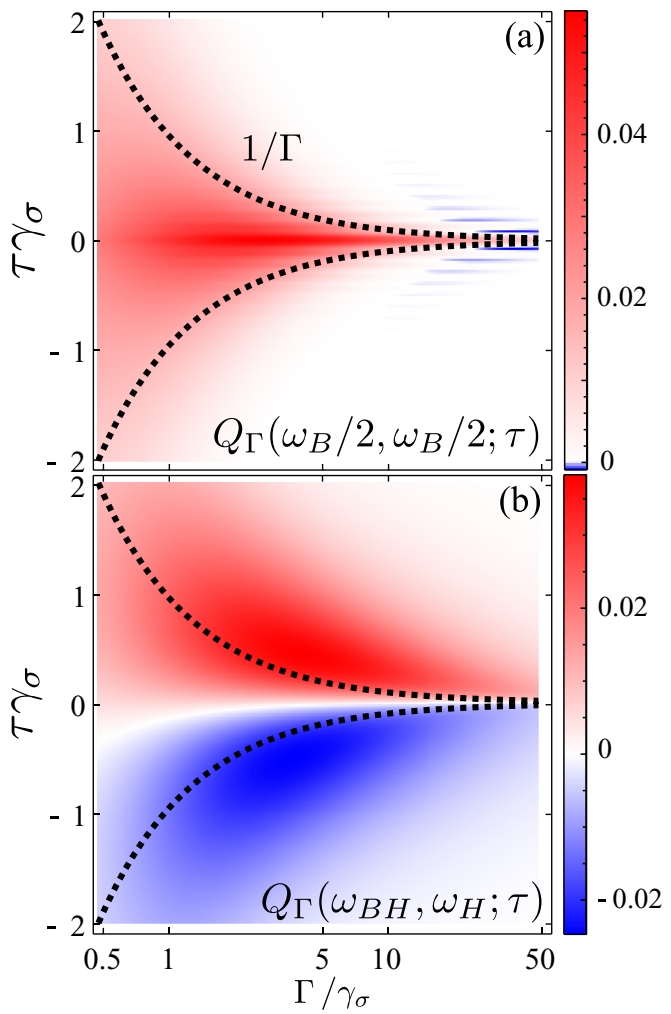

FIG. 7. (Color online) (a) Mandel parameter $Q_{\Gamma}\left(\omega_{B} / 2, \omega_{B} / 2 ; \tau\right)$ when filtering the leapfrog processes of the biexciton-exciton cascade and (b) $Q_{\Gamma}\left(\omega_{B H}, \omega_{H} ; \tau\right)$ when filtering the two peaks as a function of correlation time $\tau$ and filter linewidth $\Gamma$. This highlights again the difference between real and virtual processes. An optimum value of the filter linewidth can be found for the case of real-state transitions. Parameters are the same as in Fig. 2.

also strongly decreases and is eventually surrounded by antibunching oscillations in $\tau$, which correspond to the fast off-resonance one-photon transitions. For the set of parameters chosen here, the optimal correlations are found at $\tau=0$ for $\Gamma \sim 3 \gamma_{\sigma}$. Also, as discussed previously, in the consecutive one-photon cascade in Fig. 7(b), the temporal shape exhibits a typical asymmetric bunching or antibunching shape. The pattern is fairly robust but can, indeed, be magnified by the appropriate choice of filters (we consider here symmetric filters for simplicity of comparison). The temporal decay occurs this time approximately within a time scale of $1 / \gamma_{\sigma}$, while the maximum value for the correlations, both bunching and antibunching, is obtained at $\tau \sim 1 / \Gamma$. In this case, the maximum is found at $\Gamma \sim 3 \gamma_{\sigma}$ and $\tau \sim 1 / \Gamma$. Note that correlations are optimized for filters with a width equal to the spectral peaks ( $3 \gamma_{\sigma}$ for our choice of parameters).

\section{SUMMARY AND CONCLUSIONS}

Summing up, filtering the photons emitted by a quantum source has a dramatic impact on their correlations. Strong correlations are often found in regions of the spectrum where there is a weak emission, making their experimental detection particularly difficult since this implies coincidences of rare events. We have introduced a frequency-resolved Mandel parameter as well as a quantitative estimate of the time required to accumulate a given number of coincidences to address this problem for several paradigmatic nonlinear quantum systems. We have shown the considerable flexibility opened by frequency filtering, either in energy or in time, with possibilities to enhance correlations by varying filter linewidths (possibly asymmetrically), temporal windows of detections, and system parameters (such as pumping). Depending on whether the correlations originate from real-state transitions or involve virtual processes, different strategies should be adapted, corresponding to their intrinsically different character. With the recent experimental demonstration [29] of the underlying physics discussed here, the field of two-photon spectroscopy is now ripe to power applications and optimize resources based on applying such ideas and techniques.

\section{ACKNOWLEDGMENTS}

This work has been funded by ERC Grant POLAFLOW. E.d.V. acknowledges support from IEF project SQUIRREL (623708), and A.G.-T. acknowledges support from the Alexander Von Humboldt Foundation, IEF project NANOQUIS (625955), and the EU integrated project SIQS.
[1] R. J. Glauber, Photon correlations, Phys. Rev. Lett. 10, 84 (1963).

[2] R. J. Glauber, The quantum theory of optical coherence, Phys. Rev. 130, 2529 (1963).

[3] R. J. Glauber, Nobel lecture: One hundred years of light quanta, Rev. Mod. Phys. 78, 1267 (2006).

[4] B. Lounis and M. Orrit, Single-photon sources, Rep. Prog. Phys. 68, 1129 (2005).

[5] R. Hanbury Brown and R. Q. Twiss, A test of a new type of stellar interferometer on Sirius, Nature (London) 178, 1046 (1956).

[6] J. Wiersig, C. Gies, F. Jahnke, M. Aßmann, T. Berstermann, M. Bayer, C. Kistner, S. Reitzenstein, C. Schneider, S. Höfling, A. Forchel, C. Kruse, J. Kalden, and D. Homme, Direct observation of correlations between individual photon emission events of a microcavity laser, Nature (London) 460, 245 (2009).
[7] C. Cohen-Tannoudji and S. Reynaud, Atoms in strong lightfields: Photon antibunching in single atom fluorescence, Philos. Trans. R. Soc. London, Ser. A 293, 223 (1979).

[8] J. Dalibard and S. Reynaud, Correlation signals in resonance fluorescence: Interpretation via photon scattering amplitudes, J. Phys. (Paris) 44, 1337 (1983).

[9] N. Akopian, N. H. Lindner, E. Poem, Y. Berlatzky, J. Avron, D. Gershoni, B. D. Gerardot, and P. M. Petroff, Entangled photon pairs from semiconductor quantum dots, Phys. Rev. Lett. 96, 130501 (2006).

[10] K. Hennessy, A. Badolato, M. Winger, D. Gerace, M. Atature, S. Gulde, S. Fălt, E. L. Hu, and A. Imamoğlu, Quantum nature of a strongly coupled single quantum dot-cavity system, Nature (London) 445, 896 (2007). 
[11] M. Kaniber, A. Laucht, A. Neumann, J. M. Villas-Bôas, M. Bichler, M.-C. Amann, and J. J. Finley, Investigation of the nonresonant dot-cavity coupling in two-dimensional photonic crystal nanocavities, Phys. Rev. B 77, 161303(R) (2008).

[12] G. Sallen, A. Tribu, T. Aichele, R. André, L. Besombes, C. Bougerol, M. Richard, S. Tatarenko, K. Kheng, and J.-Ph. Poizat, Subnanosecond spectral diffusion measurement using photon correlation, Nat. Photonics 4, 696 (2010).

[13] A. Ulhaq, S. Weiler, S. M. Ulrich, R. Roßbach, M. Jetter, and P. Michler, Cascaded single-photon emission from the Mollow triplet sidebands of a quantum dot, Nat. Photonics 6, 238 (2012).

[14] B. Silva, A. González Tudela, C. Sánchez Muñoz, D. Ballarini, G. Gigli, K. W. West, L. Pfeiffer, E. del Valle, D. Sanvitto, and F. P. Laussy, Measuring photon correlations simultaneously in time and frequency, arXiv:1406.0964.

[15] L. Knöll and G. Weber, Theory of $n$-fold time-resolved correlation spectroscopy and its application to resonance fluorescence radiatio, J. Phys. B 19, 2817 (1986).

[16] G. Nienhuis, Spectral correlations in resonance fluorescence, Phys. Rev. A 47, 510 (1993).

[17] E. del Valle, A. Gonzalez-Tudela, F. P. Laussy, C. Tejedor, and M. J. Hartmann, Theory of frequency-filtered and time-resolved n-photon correlations, Phys. Rev. Lett. 109, 183601 (2012).

[18] E. del Valle, Distilling one, two and entangled pairs of photons from a quantum dot with cavity QED effects and spectral filtering, New J. Phys. 15, 025019 (2013).

[19] A. Gonzalez-Tudela, F. P. Laussy, C. Tejedor, M. J. Hartmann, and E. del Valle, Two-photon spectra of quantum emitters, New J. Phys. 15, 033036 (2013).

[20] G. Nardin, T. M. Autry, K. L. Silverman, and S. T. Cundiff, Multidimensional coherent photocurrent spectroscopy of a semiconductor nanostructure, Opt. Express 21, 28617 (2013).

[21] Y.-S. Ra, M. C. Tichy, H.-T. Lim, O. Kwon, F. Mintert, A. Buchleitner, and Y.-H. Kim, Observation of detection-dependent multi-photon coherence times, Nat. Commun. 4, 2451 (2013).

[22] G. Nardin, G. Moody, R. Singh, T. M. Autry, H. Li, F. MorierGenoud, and S. T. Cundiff, Coherent excitonic coupling in an asymmetric double ingaas quantum well arises from many-body effects, Phys. Rev. Lett. 112, 046402 (2014).

[23] M. Gessner, F. Schlawin, H. Hoffner, S. Mukamel, and A. Buchleitner, Nonlinear spectroscopy of controllable many-body quantum systems, New J. Phys. 16, 092001 (2014).

[24] F. Schlawin, M. Gessner, S. Mukamel, and A. Buchleitner, Nonlinear spectroscopy of trapped ions, Phys. Rev. A 90, 023603 (2014).

[25] C. Sánchez Muñoz, E. del Valle, C. Tejedor, and F. P. Laussy, Violation of classical inequalities by photon frequency filtering, Phys. Rev. A 90, 052111 (2014).

[26] H. Flayac and V. Savona, Heralded preparation and readout of entangled phonons in a photonic crystal cavity, Phys. Rev. Lett. 113, 143603 (2014).

[27] P. Grünwald, D. Vasylyev, J. Häggblad, and W. Vogel, Quantum measurement of broadband nonclassical light fields, Phys. Rev. A 91, 013816 (2015).

[28] R. Folman, Anomalous second order coherence and $g^{(2)}$ complementarity, arXiv:1305.3083.

[29] M. Peiris, B. Petrak, K. Konthasinghe, Y. Yu, Z. C. Niu, and A. Muller, Two-color photon correlations of the light scattered by a quantum dot, arXiv:1501.00898.
[30] B. R. Mollow, Power spectrum of light scattered by two-level systems, Phys. Rev. 188, 1969 (1969).

[31] C. Sanchez Muñoz, E. del Valle, A. González Tudela, K. Müller, S. Lichtmannecker, M. Kaniber, C. Tejedor, J. J. Finley, and F. P. Laussy, Emitters of $N$-photon bundles, Nat. Photonics 8, 550 (2014).

[32] L. Mandel and E. Wolf, Coherence properties of optical fields, Rev. Mod. Phys. 37, 231 (1965).

[33] Z. Deutsch, O. Schwartz, R. Tenne, R. Popovitz-Biro, and D. Oron, Two-color antibunching from band-gap engineered colloidal semiconductor nanocrystals, Nano Lett. 12, 2948 (2012).

[34] E. del Valle, F. P. Laussy, and C. Tejedor, Luminescence spectra of quantum dots in microcavities. II. Fermions, Phys. Rev. B 79, 235326 (2009).

[35] A. V. Poshakinskiy and A. N. Poddubny, Time-dependent photon correlations for incoherently pumped quantum dot strongly coupled to the cavity mode, J. Exp. Theor. Phys. 118, 205 (2014).

[36] H. S. Nguyen, G. Sallen, C. Voisin, Ph. Roussignol, C. Diederichs, and G. Cassabois, Ultra-coherent single photon source, Appl. Phys. Lett. 99, 261904 (2011).

[37] C. Matthiesen, A. N. Vamivakas, and M. Atatüre, Subnatural linewidth single photons from a quantum dot, Phys. Rev. Lett. 108, 093602 (2012).

[38] Y.-M. He, Y. He, Y.-J. Wei, D. Wu, M. Atatüre, C. Schneider, S. Höfling, M. Kamp, C.-Y. Lu, and J.-W. Pan, On-demand semiconductor single-photon source with near-unity indistinguishability, Nat. Nanotechnol. 8, 213 (2013).

[39] M. N. Makhonin, J. E. Dixon, R. J. Coles, B. Royall, I. J. Luxmoore, E. Clarke, M. Hugues, M. S. Skolnick, and A. M. Fox, Waveguide coupled resonance fluorescence from on-chip quantum emitter, Nano Lett. 14, 6997 (2014).

[40] M. N. Makhonin, J. E. Dixon, R. J. Coles, B. Royall, E. Clarke, M. S. Skolnick, and A. M. Fox, On-chip resonantly-driven quantum emitter with enhanced coherence, arXiv:1404.3967.

[41] K. Wódkiewicz, Laser linewidth effects in intensity correlations in resonance fluorescence, Phys. Lett. A 77, 315 (1980).

[42] H. F. Arnoldus and G. Nienhuis, Collisional redistribution of intense phase-fluctuation radiation, J. Phys. B 16, 2325 (1983).

[43] H. F. Arnoldus and G. Nienhuis, Photon correlations between the lines in the spectrum of resonance fluorescence, J. Phys. B 17, 963 (1984).

[44] V. N. Shatokhin and S. Ya. Kilin, Correlation measurements in resonance fluorescence with spectral resolution and atomic inversion via detection of a spectrally filtered photon, Phys. Rev. A 63, 023803 (2001).

[45] A. Aspect, G. Roger, S. Reynaud, J. Dalibard, and C. CohenTannoudji, Time correlations between the two sidebands of the resonance fluorescence triplet, Phys. Rev. Lett. 45, 617 (1980).

[46] S. Weiler, D. Stojanovic, S. M. Ulrich, M. Jetter, and P. Michler, Postselected indistinguishable single-photon emission from the mollow triplet sidebands of a resonantly excited quantum dot, Phys. Rev. B 87, 241302(R) (2013).

[47] E. del Valle, S. Zippilli, F. P. Laussy, A. Gonzalez-Tudela, G. Morigi, and C. Tejedor, Two-photon lasing by a single quantum dot in a high- $Q$ microcavity, Phys. Rev. B 81, 035302 (2010). 
[48] E. del Valle, A. Gonzalez-Tudela, E. Cancellieri, F. P. Laussy, and C. Tejedor, Generation of a two-photon state from a quantum dot in a microcavity, New J. Phys. 13, 113014 (2011).

[49] G. Chen, T. H. Stievater, E. T. Batteh, X. Li, D. G. Steel, D. Gammon, D. S. Katzer, D. Park, and L. J. Sham, Biexciton quantum coherence in a single quantum dot, Phys. Rev. Lett. 88, 117901 (2002).
[50] I. A. Akimov, J. T. Andrews, and F. Henneberger, Stimulated emission from the biexciton in a single selfassembled II-VI quantum dot, Phys. Rev. Lett. 96, 067401 (2006).

[51] Y. Ota, S. Iwamoto, N. Kumagai, and Y. Arakawa, Spontaneous two-photon emission from a single quantum dot, Phys. Rev. Lett. 107, 233602 (2011). 